\title{
Design of keystream Generator utilizing Firefly Algorithm
}

\author{
Mohammed Salih Mahdi \\ University of Information Technology \\ and Communications \\ BIT Dept., Business Information College \\ Baghdad, Iraq \\ mohammed.salih@uoitc.edu.iq
}

Recived : $1 \backslash 8 \backslash 2018$

Revised : $619 \backslash 2018$

\author{
Nidaa Flaih Hassan
}

University of Technology

Computer science Dept

Baghdad, Iraq

110020@uotechnology.edu.iq

Available online : 26/9/2018

DOI: $10.29304 / j q c m .2018 .10 .3 .441$

\begin{abstract}
:
Stream cipher is one of encryption procedures for sending data in internet; stream cipher is suitable in telecommunications and real-time apps. The robustness measurement of stream cipher is according to the randomness of keystream that is utilized. If the random series of keystream generator is low, the keystream of stream cipher can be read and encrypted data by stream cipher become vulnerable to attackers. This paper utilizes Firefly Algorithm based Local Key Generation for generation keystream. The generated keystream is independent of original messages. The randomness of keystream series of Firefly passing the five standard criteria. The suggested keystream generator is wordestablished appropriated to fast real-time apps than are bit-established linear stream ciphers. Furthermore, the suggested keystream generator satisfies the three demands of benchmarks such as maximum correlation, robust randomness and huge complexity.
\end{abstract}

Key Words. Stream Cipher, keystream Generator, Firefly Algorithm, Local Keys Function. 


\section{Introduction}

Cryptography is an exercise together with analyze procedures for safe communication in the presence of adversaries. Generally, it is about founding and studying protocols which overcome the technique of attackers and that are linked to a diversity of parts in data security [1]. The mammoth issues of secure telecommunication is keeping the confidential information from interception. In cipher frameworks, It is popular that the experts of encryption procedures required techniques to discover an orderly procedure in checking their ciphers to guarantee that they are protected from attackers [2][3]. One of a popular symmetric procedure is stream cipher, all portion of original message and keystream are encrypted together. The keys of Stream cipher utilized for encryption procedure is altered randomly. Consequently, the cipher that's created is mathematically very hard to breach. The altering of random keystream will not permit any sample to be frequent that allocate a guide to attacker to breach cipher data [4], [5].

Stream cipher is suitable on equipment and programs, and in some situations obligatory in telecommunications and real-time apps chiefly with restricted memory [6], [7]. Stream cipher is less apt to cryptanalysis due to identical portions of original messages are encipher with various portions of the keystream [8]. The essential idea of stream cipher is one-time pad cipher which is refer to Vernam cipher, necessity that is true random series with generated keystream, Both transmitter and recipient are participated keystream, and keystream only utilized once [9]. The cons of stream cipher are the total volume of the keystream and the original message should be the identical. Subsequently a huge quantity of keystream have to be kept and transmitted. Furthermore, if the random series is discover, the keystream utilized for encryption procedure can be keep track readily [10].

Many different papers utilized to evolve Stream Cipher generator for Encryption according to SI and several techniques, In[11] suggested keystream procedure for encryption data according to Ant Colony and the allocation of letters in the original message. In [12] suggested keystream generator according to a Particle swarm optimization for encryption data. The con of these articles is that enumeration the appearance of letters of keystream in the original messages. Of course, that it excess the overall time consumption for encryption, when the volume of keystream selected is huge.
In [13] suggested keystream generator according to 3D chaotic maps and Particle swarm optimization to create a random number generator and tested by five standard criteria. In [14] suggested keystream generator according to on dynamic the thought of updates composite LFSR stream cipher every update in message that indicates to has a huge complexity encryption procedure and tested by standard criteria

Concerning, the stream cipher and Firefly Algorithm that is debated at the previously, the contribution of this paper is suggested Keystream generator utilizing Firefly Algorithm based Local Key Generation where the generated keystream is independent of original messages .

\section{Firefly Algorithm}

Optimization is an arithmetic procedure for getting either highest or smallest value of a target function by selecting a best solution from a set of solutions [15]. There are a huge number of optimization problems, which were complicated and toughed to discover a solution at a sensible time in different areas like business, operations research and computer science [16].

These optimization problems can be successfully solved by applied Biology-NatureInspired-Metaheuristic-Algorithms (BNIMAs) mostly that according to swarm intelligence (SI) which is a subset of Artificial Intelligence. Birds, Wasps, Ants, Bugs ,Bees and Firefly are different models of the family of BNIMAs which are using the behavior of SI based on target function [17][18].

In optimization problems, firefly algorithm is a one of SI clans of algorithms that lately exhibited magnificent performances by presenting best solutions [19] as shown in Algorithm (1). Firefly algorithm utilizes the subsequent three essentials [20][21]:

- All firefly would be going to other fireflies that have a high attractiveness irrespective of their gender.

- The attraction of a firefly is apt to its lighting that is minify as distance of different firefly rises. Fireflies shall move arbitrarily, when no it's lighter one than a specific firefly.

- The aim of target function is locating the illumination of firefly.

Three critical criteria in Firefly algorithm can be summarized as [22]: 
- The $1^{\text {st }}$ critical criteria is attraction (attractiveness $\mathrm{N}$ ) that is specified by its lighting strength as Eq.1 follows:

$\mathrm{N}=\mathrm{N}_{0} e^{-v t^{2}}$

$\mathrm{N}_{0}$ is an attraction at distance $\mathrm{t}$ equal to zero, while $\mathrm{v}$ is refer to lighting absorption in weather.

- The $2^{\text {nd }}$ critical criteria is distance that is specified by utilizing the Cartesian distance between two fireflies as Eq.2 follows:

$\mathrm{R}_{\mathrm{i}, \mathrm{j}}=\sqrt{\sum_{S=1}^{n}\left(F_{I, S}-F_{J, S}\right)^{2}}$

In the n-dimensional space, $F_{i, s}$ is $\mathrm{s}^{\text {th }}$ component of coordinate $F_{i}$ of $\mathrm{i}^{\text {th }}$ Firefly.

- The $3^{\text {rd }}$ critical criteria is movement that is specified by brightness for instance, firefly (a) would be going to firefly (b) that has a high attractiveness as Eq.3 follows:

$\mathrm{M}_{\mathrm{a}}=\mathrm{M}_{\mathrm{a}}+\mathrm{N}_{0} e^{-v R^{2} i j}\left(\mathrm{M}_{\mathrm{b}-} \mathrm{M}_{\mathrm{a}}\right)+\mathrm{P}(\mathrm{r}-0.4) \quad$ Eq. 3 Where $\mathrm{P}$ is refer to randomization value, $\mathrm{r}$ is refer to a random value that its range between 0 and 1 a, $M_{a}$ is refer to movement firefly (a) and $M_{b}$ is refer to movement firefly (b). Furthermore Firefly is equal to particle swarm optimization when $\mathrm{v}$ is equal to zero [22].

\section{Algorithm (1): Firefly Algorithm}

Input: number of firefly population ,light absorption coefficient v, max- iteration, objective

\section{Output: set of keystream}

\section{Begin}

Step $_{1}$ : Determine the objective function.

Step $_{2}$ : Generate initial population of fireflies.

Step $_{3}$ : Calculate the Light intensity at fireflies based on objective function.

Step $_{4}$ : iteration $=1$

Step $_{5}: A=1, B=1$

Step $_{6}$ : if fitness function of firefly (A) less than fitness function of firefly (B) then Move firefly (A) towards firefly (B)

Step $_{7}$ : modify attraction differs with distance through $e^{-v t^{2}}$

Step $_{8}$ : Evaluate modern resolutions and modify brightness intensity.

Step $_{9}: B=B+1$

Step $_{10}$ : if (B less than or equal to number of fireflies) Goto $\mathrm{Step}_{6}$

Step $_{11}: A=A+1, B=1$

Step $_{12}$ : if (A less than or equal to number of fireflies) Goto Step 6

$\mathrm{Step}_{13}$ : Rank fireflies and find the current best

Step $_{14}$ : iteration $=$ iteration +1

Step $_{15}$ : if (iteration less than or equal to maxiteration) Goto Step $_{5}$

End

\section{Methodology of Designing Stream Cipher utilizing Firefly Algorithm}

The main essentials of Firefly Algorithm and stream ciphers have been learned and analyzed. In this Methodology, Firefly Algorithm based Local Key Generation (FAbLKG) is suggested for generation keystreams as exhibited in Algorithm (2). In FAbLKG, a Firefly is utilized to assign a keystream. Each Firefly have many keys inside that keystream, the keys in the keystream are set of bits can be 0 or 1 . For instance, if the length of keys of each Firefly is equal to 512 consequently it is depicted by Eq. 4 .

Firefly (keystream) key1, key2 ,. , key512 Eq. 4

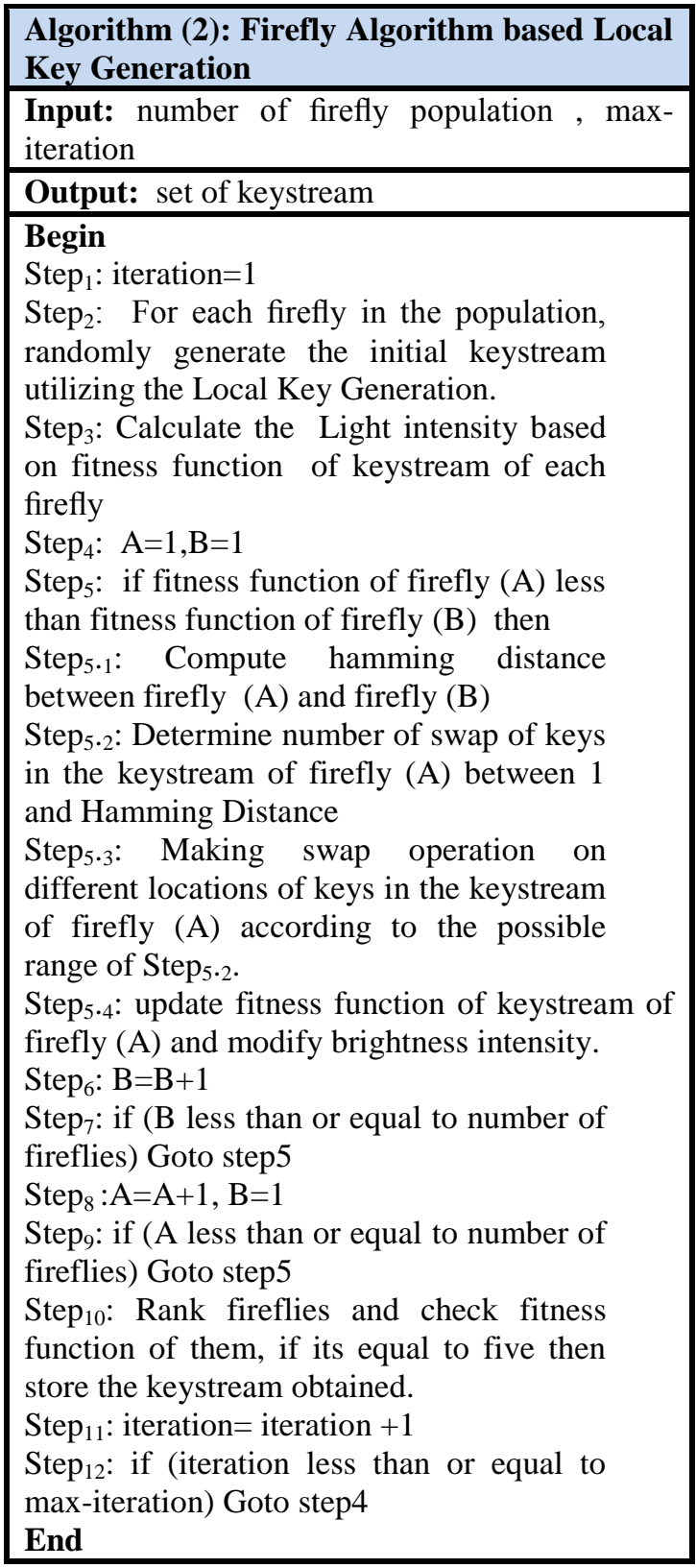




\subsection{Representation of Fireflies}

For generating a keystream, Firefly Algorithm utilize the binary code as a solution. The Firefly Algorithm initiates with an elementary population encompasses set of fireflies (keystream) according to Local Key Generation.

\subsubsection{Local Key Generation}

Local Key Generation (LKG) is a novel word-established NLFSR stream ciphers which is offer numerous volumes of keystream per round than bit-established LFSRs stream ciphers, according to the word volume. Thus, in LKG an equilibrium is accomplished between security and efficiency. LKG can be referred as a development of the outcome feedback mode. The outcome of keystream is furthermore the feedback to the inner state. LKG has initially seed of 512 bits inner state sets by initialization vector. These bits are splitted into sixteen 32-bit words categorized $\mathrm{W}_{1}$ to $\mathrm{W}_{16}$ and making XOR and Local Function feeding among some of them as clarified in Figure (1).

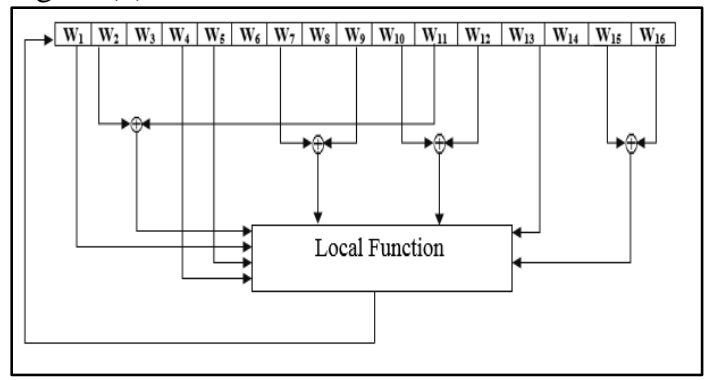

Figure (1): Local Key Generation

The Local Function that is called on one occasion per iteration, impacts the inner state to produce 512 bits of keystream per iteration. The input of Local Function is 512 bits (eight variable, the size of each variable is 32 bits). In Local Function, A network of summation mod $2^{32}$ and XOR are utilized for diffusion and it conclude of three procedures as clarified in algorithm (3). The $1^{\text {st }}$ procedure of Local Function is Pre-confusion. The $2^{\text {nd }}$ procedure of Local Function is combination ( $\mathrm{M}$ and $\mathrm{Q}$ Functions) according to two s-box $\left(\mathrm{O}_{1}\right.$ and $\left.\mathrm{O}_{2}\right)$ as clarified in algorithm (4). The $3^{\text {rd }}$ procedure of Local Function is Post-confusion.

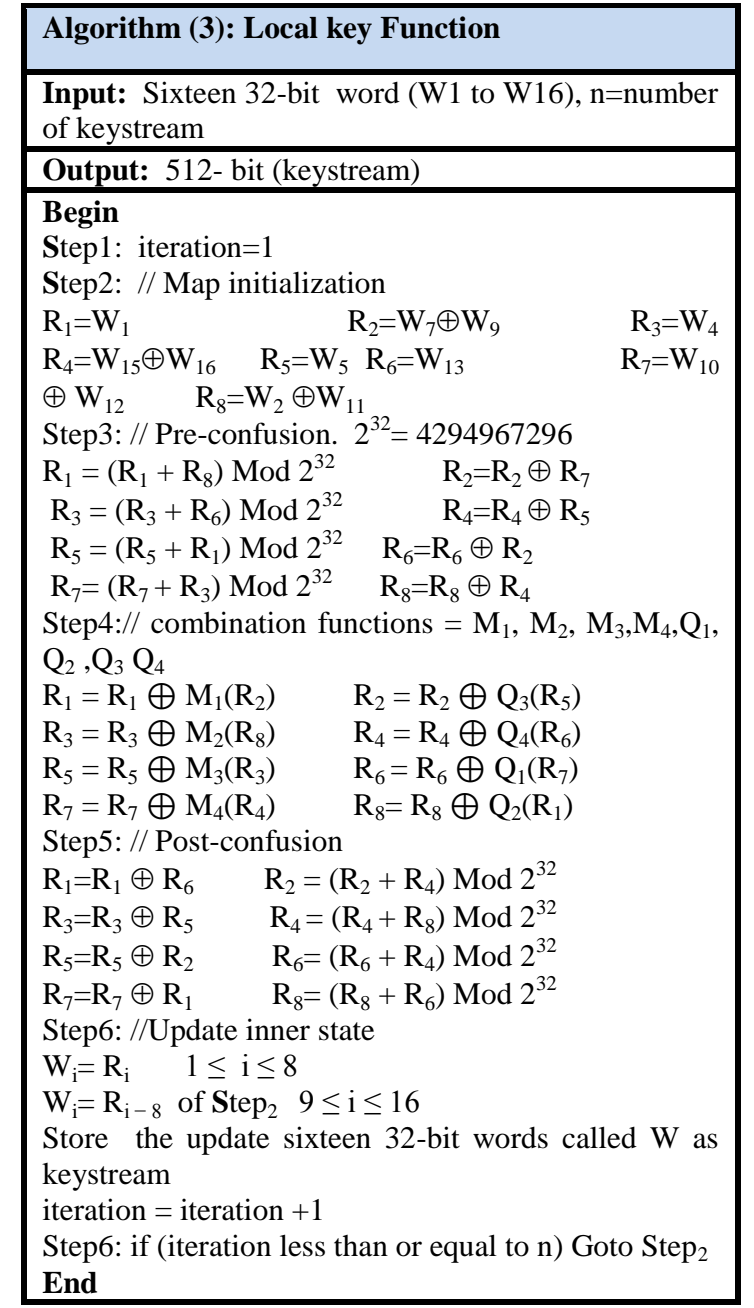

\begin{tabular}{|l|}
\hline Algorithm (4): $M$ and Q Functions \\
\hline Input: 32-bit , N=number of $\mathrm{M}$ and $\mathrm{Q}$ function, $\mathrm{O} 1=$ \\
$32 \times 32$ and $\mathrm{O} 2=32 \times 32$ \\
\hline Output: Update 32-bit \\
\hline Begin \\
Step1: Break 32 -bit to four variable $\left(\mathrm{y}_{1}, \mathrm{y}_{2}, \mathrm{y}_{3}, \mathrm{y}_{4}\right)$ \\
Step2: \\
if $(\mathrm{N}==1)$ then \\
$\mathrm{M}_{1}(\mathrm{y})=\mathrm{O}_{1}\left(\mathrm{y}_{1}\right) \oplus \mathrm{O}_{1}\left(\mathrm{y}_{2}\right) \oplus \mathrm{O}_{1}\left(\mathrm{y}_{3}\right) \oplus \mathrm{O}_{2}\left(\mathrm{y}_{4}\right)$ \\
$\mathrm{Q}_{1}(\mathrm{y})=\mathrm{O}_{2}\left(\mathrm{y}_{1}\right) \oplus \mathrm{O}_{2}\left(\mathrm{y}_{2}\right) \oplus \mathrm{O}_{2}\left(\mathrm{y}_{3}\right) \oplus \mathrm{O}_{1}\left(\mathrm{y}_{4}\right)$ \\
$\quad$ Else if $(\mathrm{N}==2)$ then \\
$\mathrm{M}_{2}(\mathrm{y})=\mathrm{O}_{1}\left(\mathrm{y}_{1}\right) \oplus \mathrm{O}_{1}\left(\mathrm{y}_{2}\right) \oplus \mathrm{O}_{2}\left(\mathrm{y}_{3}\right) \oplus \mathrm{O}_{1}\left(\mathrm{y}_{4}\right)$ \\
$\mathrm{Q}_{2}(\mathrm{y})=\mathrm{O}_{2}\left(\mathrm{y}_{1}\right) \oplus \mathrm{O}_{2}\left(\mathrm{y}_{2}\right) \oplus \mathrm{O}_{1}\left(\mathrm{y}_{3}\right) \oplus \mathrm{O}_{2}\left(\mathrm{y}_{4}\right)$ \\
$\quad$ Else if $(\mathrm{N}==3)$ then \\
$\mathrm{M}_{3}(\mathrm{y})=\mathrm{O}_{1}\left(\mathrm{y}_{1}\right) \oplus \mathrm{O}_{2}\left(\mathrm{y}_{2}\right) \oplus \mathrm{O}_{1}\left(\mathrm{y}_{3}\right) \oplus \mathrm{O}_{1}\left(\mathrm{y}_{4}\right)$ \\
$\mathrm{Q}_{3}(\mathrm{y})=\mathrm{O}_{2}\left(\mathrm{y}_{1}\right) \oplus \mathrm{O}_{1}\left(\mathrm{y}_{2}\right) \oplus \mathrm{O}_{2}\left(\mathrm{y}_{3}\right) \oplus \mathrm{O}_{2}\left(\mathrm{y}_{4}\right)$ \\
$\quad$ Else \\
$\mathrm{M}_{4}(\mathrm{y})=\mathrm{O}_{2}\left(\mathrm{y}_{1}\right) \oplus \mathrm{O}_{1}\left(\mathrm{y}_{2}\right) \oplus \mathrm{O}_{1}\left(\mathrm{y}_{3}\right) \oplus \mathrm{O}_{1}\left(\mathrm{y}_{4}\right)$ \\
$\mathrm{Q}_{4}(\mathrm{y})=\mathrm{O}_{1}\left(\mathrm{y}_{1}\right) \oplus \mathrm{O}_{2}\left(\mathrm{y}_{2}\right) \oplus \mathrm{O}_{2}\left(\mathrm{y}_{3}\right) \oplus \mathrm{O}_{2}\left(\mathrm{y}_{4}\right)$ \\
End if \\
End
\end{tabular}




\subsection{Fitness Function of Fireflies}

For each firefly, the fitness function is utilize to measure the robustness of keystream as brightness (Light Intensity) which is computed by testing the five standard criteria of generated keystream as interpreted in Table (1). If the generated keystream is exceeding all five standard criteria therefore robustness of Firefly (keystream) is five and if the generated keystream is exceeding four of standard criteria therefore robustness of Firefly is four and so on.

\subsection{Moving of Fireflies}

The Firefly Algorithm initiates with an elementary population randomly according to Local Key Generation consequently the fireflies dispersed in state space. If the robustness of keystream (lighting) of Firefly (A) less than the firefly(B) consequently Firefly (A) move in the direction of the Firefly (B) by swapping the keys in the keystream of the Firefly (A). The range of swapping keys in the keystream of Firefly (A) is determined randomly from one to Hamming Distance between Firefly (A) and Firefly (B) as depicted by Eq2. Indeed, the swapping keys in the keystream creates modern diffusions (permutations). For example, assume the fitness (robustness of keystream) of Firefly (A) and Firefly (B) are 2 and 4 respectively, and the Hamming Distance between Firefly (A) and Firefly (B) is 5. Consequently, Firefly (A) move in the direction of the Firefly (B) by swapping keys in the keystream of the Firefly (A). The range of swapping keys in the keystream of Firefly (A) is randomly selected between 1 and 5 (Hamming Distance). Assume the selected the range of swap is 3 that is led to making at most three swap of keys (different swap locations) in the keystream of Firefly (A).

Table (1): Five Standard Criteria Equations Information [23]

\begin{tabular}{|c|c|}
\hline Five Standard Criteria Equations & Information on Five Standard Criteria \\
\hline$T 1=\frac{(M 0-M 1)^{2}}{M}$ & $\begin{array}{l}\text { M0: number of 0's in keystream. } \\
\text { M1: number of 1's in keystream. } \\
\text { M: total size of keystream. }\end{array}$ \\
\hline $\begin{aligned} T 2=\frac{4}{M-1}( & (M 11)^{2}+(M 00)^{2} \\
& \left.+(M 01)^{2}+(M 10)^{2}\right) \\
& -\frac{2}{M}\left(M 1^{2}+M 0^{2}\right)+1\end{aligned}$ & $\begin{array}{l}\text { M11: number of } 11 \text { 's in keystream. } \\
\text { M00: number of } 00 \text { 's in keystream. } \\
\text { M01: number of } 01 \text { 's in keystream. } \\
\text { M10: number of } 10 \text { 's in keystream }\end{array}$ \\
\hline$T 3=\frac{2^{\mathrm{N}}}{P}\left(\sum_{j=1}^{2^{\mathrm{N}}} M_{j}^{2}\right)-\mathrm{P}$ & $\begin{array}{l}M_{j} \text { : number of appearance of the } \mathrm{j}^{\text {th }} \text { of length } \mathrm{N} P=\frac{\mathrm{M}}{N} \\
\qquad \frac{\mathrm{M}}{\mathrm{N}} \geq\left(5 * 2^{\mathrm{N}}\right)\end{array}$ \\
\hline $\begin{aligned} T 4=\left(\sum_{j=1}^{N} \frac{\left(B_{j}-P_{j}\right)^{2}}{P_{j}}\right) & \\
& +\left(\sum_{j=1}^{N} \frac{\left(G_{j}-P_{j}\right)^{2}}{P_{j}}\right)\end{aligned}$ & $\begin{array}{l}\mathrm{N} \text { :maximum } \mathrm{j} \text { for which } P_{j} \geq 5 \text {. } \\
B_{j} \text { : Amount of blocks (subsequences runs of } 1 \text { 's) of } \\
\text { length } \mathrm{j} \text { in M. } \\
G_{j} \text { : amount of gabs (subsequences runs of } 0 \text { 's) of length } \\
\mathrm{j} \text { in M. } \\
\qquad P_{j}=\frac{\mathrm{M}-\mathrm{j}+3}{2^{(\mathrm{j}+2)}}\end{array}$ \\
\hline $\mathrm{T} 5=\frac{2\left(\mathrm{~A}(\mathrm{k})-\frac{(\mathrm{M}-\mathrm{k})}{2}\right)}{\sqrt{(\mathrm{M}-\mathrm{k})}}$ & $\begin{array}{r}\mathrm{k}: 1 \leq \mathrm{k} \leq[\mathrm{m} / 2] \\
\mathrm{A}(\mathrm{k})=\sum_{\mathrm{j}=0}^{\mathrm{M}-\mathrm{k}-1}\left(\mathrm{~S}_{\mathrm{j}}+\mathrm{S}_{+\mathrm{k}}\right) \operatorname{Mod} 2\end{array}$ \\
\hline
\end{tabular}




\section{Case Study of Suggested \\ Keystream Generator}

Suppose, the number of firefly population is two and max- iteration is one. The following steps illustrated the suggested keystream generator according to Algorithm (2), Algorithm (3) and Algorithm (4).

- $\mathrm{S}_{1}$ : Suppose the seed of two fireflies:

Firefly(A) $\rightarrow$ "5d413d691dd67b3449bf371ac15968af eebe6361f83cf5c359ae7fc83178ab8ce0157eba9a3 8655da4abe24f359f664ad6036972c002764f33738 94d49a6df77"

Firefly $(B) \rightarrow " 27 d 591 \mathrm{c} 45 \mathrm{f} 02 \mathrm{e} 8 \mathrm{c} 6 \mathrm{~d} 0624 \mathrm{a} 6 \mathrm{a} 20 \mathrm{~d} 9 \mathrm{~b} 4 \mathrm{bb}$ 5f59a6e8e497a2e235feb4ad5d45ec8aca348c8f24a 269ae9c608d099a652cd973f9098e69360fab3c318 $80567 \mathrm{ca} 82 \mathrm{e} 4 "$.

Each one of fireflies is represented by 128 hexanumber that is equal to 512 bits.

- $\mathrm{S}_{2}$ : At first, each firefly is splitted to sixteen 8hexa-number $\left(\mathrm{W}_{1}\right.$ to $\left.\mathrm{W}_{16}\right)$, for instance Firefly(A) is splitted to $\left(\mathrm{W}_{1}=\quad\right.$ "5d413d69", $\mathrm{W}_{2}=$ "1dd67b34",.., $\mathrm{W}_{16}=$ "49a6df77").

- $\mathrm{S}_{3}$ : For Map initialization of keystream for Local key Function utilized according to $\mathbf{S t e p}_{2}$ of Algorithm (3), $\mathrm{R}_{1}=\mathrm{W}_{1} \rightarrow \mathrm{R}_{1}=$ "5d413d69" $\rightarrow \mathrm{R}_{2}=$ $\mathrm{W}_{7} \oplus \quad \mathrm{W}_{9} \rightarrow \mathrm{R}_{2}=" 59 \mathrm{ae} 7 \mathrm{fc} 8 " \oplus$ "e0157eba"="b9bb0172" and so.

- $\mathrm{S}_{4}$ : For Pre-confusion of keystream for Local key Function utilized according to $\mathbf{S t e p}_{3}$ of Algorithm (3), $\mathrm{R}_{1}=\left(\mathrm{R}_{1}+\mathrm{R}_{8}\right)$ Mod $2^{32} \rightarrow \mathrm{R}_{1}=$ "16bed6e4", $\mathrm{R}_{2}=\mathrm{R}_{2} \oplus \mathrm{R}_{7} \rightarrow \mathrm{R} 2=" 161 \mathrm{c} 0265$ "and so.

- $\mathrm{S}_{5}$ : For combination functions of keystream for Local key Function utilized according to $\mathbf{S t e p}_{4}$ of Algorithm (3), $\mathrm{R}_{1}=\mathrm{R}_{1} \oplus \mathrm{M}_{1}\left(\mathrm{R}_{2}\right) \quad$ At first, calculating $\mathrm{M}_{1}\left(\mathrm{R}_{2}\right)$ according to Algorithm (4), $\mathrm{y}=\mathrm{R}_{2}$. y will be splitted to $\mathrm{y} 1, \mathrm{y} 2, \mathrm{y} 3$ and $\mathrm{y} 4$, considering as indexing of $\mathrm{O}_{1} ; \mathrm{O}_{2}$ (two S-box in Appendix) so, $\mathrm{M}_{1}(\mathrm{y})=\mathrm{O}_{1}\left(\mathrm{y}_{1}\right) \oplus \mathrm{O}_{1}\left(\mathrm{y}_{2}\right) \oplus \mathrm{O}_{1}\left(\mathrm{y}_{3}\right)$ $\bigoplus \mathrm{O}_{2}\left(\mathrm{y}_{4}\right) \rightarrow \mathrm{M}_{1}\left(\mathrm{R}_{2}\right)=" 5 \mathrm{ddc} 0340 "$ then make $\oplus$ with "16bed6e4" $\rightarrow \mathrm{R}_{1}=$ "5bc6f670" and so on.

- $\mathrm{S}_{6}$ : For Post-confusion of keystream for Local key Function utilized according to $\mathbf{S t e p}_{5}$ of Algorithm (3),

$\mathrm{R}_{1}=\mathrm{R}_{1} \oplus \mathrm{R}_{6} \rightarrow \mathrm{R}_{1}=" \mathrm{~b} 80 \mathrm{~d} 61 \mathrm{~b} 4 " \oplus$ "e3cb97c4"

$\rightarrow \mathrm{R}_{1}=$ "b80d61b4" and so on.
- $\mathrm{S}_{7}$ : For Update inner state of keystream for Local key Function utilized according to Step $_{6}$ of Algorithm (3), $\mathrm{W}_{\mathrm{i}}=\mathrm{R}_{\mathrm{i}}, 1 \leq \mathrm{i} \leq 8, \mathrm{~W}_{\mathrm{i}}=\mathrm{R}_{\mathrm{i}-8}$ of Step $_{2}$ of Algorithm (3), $9 \leq \mathrm{i} \leq 16$, W1="b80d61b4",W2="a71ed0fa", and so on.

- $\mathrm{S}_{8}$ : The output of local key function of two fireflies:

Firefly(A) $\rightarrow=$ "b80d61b4a71ed0fa97c5bc4c2 02b6aebfa54589b03f702af5cf8116440967f3 65d413d691dd67b3449bf371ac15968afeebe 6361f83cf5c359ae7fc83178ab8c"

Firefly(B) $\rightarrow$ "b94b365494d6e6db29b3a3d6ef a21ef6cda2534b5e42a326606cba30407549e 227d591c45f02e8c6d0624a6a20d9b4bb5f59 a6e8e497a2e235feb4ad5d45ec8a".

- $\mathrm{S}_{9}$ : for each firefly, calculating Light intensity According to five standard criteria that is illustrated in Table (1). The fitness function of firefly (A) is 5 , i.e. firefly (A) is passing five standard criteria. However, the fitness function of firefly (B) is 3,i.e. firefly (B) is failing in serial test and run test.

- $\mathrm{S}_{10}$ : According to $\mathrm{Step}_{5}$ of Algorithm (2), compute hamming distance between firefly (A) and firefly (B) by convert them to binary and count the matching number as maximum number of making swap operation on different locations.

- $\mathrm{S}_{11}$ : check fitness function of two firefly, already, firefly (A) is passing five standard criteria early then discovering firefly (B) is also passing five standard criteria too due to iterated swap processing. So the final result of two fireflies in binary form.

firefly(A)="10111000000011010110000110 110100101001110001111011010000111110 101001011111000101101111000100110000 100000001010110110101011101011111110 10010101000101100010011011000000111 110111000000101010111101011100111110 000001000101100100010000001001011001 111111001101100101110101000001001111 010110100100011101110101100111101100 110100010010011011111100110111000110 101100000101011001011010001010111111 101110101111100110001101100001111110 000011110011110101110000110101100110 101110011111111100100000110001011110 001010101110001100".

Firefly(B)="10111100010010111001011001 110100000101001101011011100011010110 110010100110110110101000110101010011 001111101000100101111111110110110010 01 


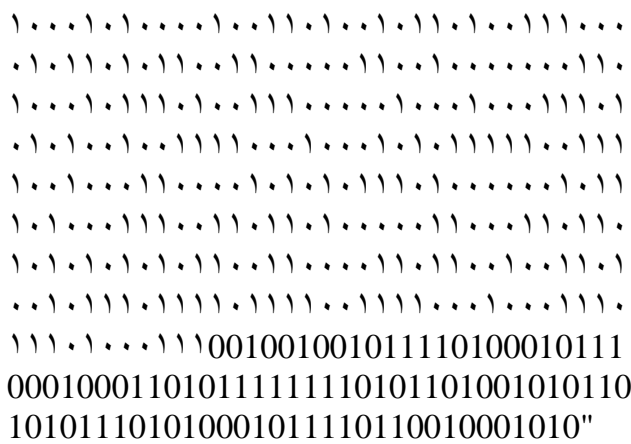

The implementation of above case study of suggested keystream Generator for generating two fireflies as two Keystream as showing in Figure (2) utilizing JavaScript language

Figure (2): Implementation of sample Case Study

\section{Results of Suggested Keystream Generator}

This paper utilizing one of SI procedures called firefly with local key generation for generation keystream. Through implementation of suggested key generator, the procedure has several variables as illustrates in Table 2.

Table 2. Variables chosen of suggested keystream generator

\begin{tabular}{|r|l|}
\hline \multicolumn{1}{|l|}{ Variables } & Range \\
\hline number of firefly population & 2 to 1000 \\
\hline max- iteration & 1 to 1000 \\
\hline \multicolumn{1}{|c|}{ Length of firefly } & 512 bits \\
\hline $\begin{array}{l}\text { Number of swap of keys } \\
\text { in the keystream of } \\
\text { firefly. }\end{array}$ & 1 to \\
& Hamming \\
\end{tabular}

If utilize only firefly algorithm for generation keys will be stuck in local optimal and some of generated keystream will be poor. While, the generated keystream of suggested FAbLKG has high randomness by passing the five-benchmark tests due to utilizing local key generation and firefly algorithm. Local key generation is prevent the firefly algorithm from stuck in local optimal and generated keystream
The suggested FAbLKG is implemented on various entire size of data utilizing JavaScript language. The FAbLKG performance is utilized on many keystream series of Firefly of length 512 bits. Each iteration, discovering the best keystream if it found then store it, when the robustness of keystream series of Firefly is five that means passing the five benchmark tests.

For instant, the result of one keystream series of Firefly that is passing the five standard criteria as interpreted in Table (3).

Table (3): Five Standard Criteria Performance

\begin{tabular}{|c|c|c|c|}
\hline $\begin{array}{c}5- \\
\text { benchmark } \\
\text { Tests }\end{array}$ & $\begin{array}{c}\text { Test } \\
\text { Value }\end{array}$ & $\begin{array}{c}\text { Threshol } \\
\mathbf{d}\end{array}$ & $\begin{array}{c}\text { Test } \\
\text { value }< \\
\text { Threshol } \\
\text { d }\end{array}$ \\
\hline Frequency T1 & 0.007 & 3.841 & pass \\
\hline Serial T2 & 4.941 & 5.991 & pass \\
\hline Poker T3 & $\begin{array}{c}11.81 \\
3\end{array}$ & 24.995 & pass \\
\hline Runs T4 & 5.028 & 12.591 & pass \\
\hline $\begin{array}{c}\text { Autocorrelatio } \\
\text { n T5 }\end{array}$ & 0.531 & 1.96 & pass \\
\hline \multicolumn{2}{|c|}{} \\
\hline
\end{tabular}




\section{Conclusion}

This paper suggests FAbLKG with robust infrastructure with more diffusion of the generated keystream, From examining the best keystream of rounds by utilizing FAbLKG and rely on randomness standard, it is simple to observe that the suggested FAbLKG have a huge keystreams solutions which are satisfied the three demands of benchmarks such as maximum correlation, robust randomness, huge complexity. Attacker want to $2^{512}$ prospective trails to breach of the generated keystream of FAbLKG, consequently in this situation, a bruteforce attacking seems unwieldy step. FAbLKG is Word-established may be better appropriated to fast real-time apps than are bit-established linear stream ciphers, FAbLKG is offer numerous volumes of keystream per iteration than bitestablished LFSRs.

\section{References}

[1] H. Feistel, "Cryptography and computer privacy," Sci. Am., vol. 228, no. 5, pp. 15-23, 1973.

[2] H. Gustafson, E. Dawson, L. Nielsen, and W. Caelli, "A computer package for measuring the strength of encryption algorithms," Comput. Secur., vol. 13, no. 8, pp. 687-697, 1994.

[3] G. J. Krishna, R. Vadlamani, and S. N. Bhattu, "Key Generation for Plain Text in Stream Cipher via BiObjective Evolutionary Computing," Appl. Soft Comput., 2018.

[4] P. P. Deepthi, D. S. John, and P. S. Sathidevi, "Design and analysis of a highly secure stream cipher based on linear feedback shift register," Comput. Electr. Eng., vol. 35, no. 2, pp. 235-243, 2009.

[5] T. Good and M. Benaissa, "Hardware results for selected stream cipher candidates," State Art Stream Ciphers, vol. 7, pp. 191-204, 2007.

[6] V. Menezes, "Oorschot:" Handbook of Applied Cryptography', 1997," CRC Press, vol. 200, pp. 610.

[7] M. Stamp, Information security: principles and practice. John Wiley \& Sons, 2011.

[8] A. AL RASEDY and A. AL SWIDI, "An advantages and Dis Advantages of Block and Stream Cipher," in Third Annual Scientific of the Faculty of basic Education Conference., 2010.

[9] M. J. B. Robshaw, "Stream ciphers," RSA Lab. Tech. Rep., 1995.

[10] C. P. Pfleeger and S. L. Pfleeger, Security in computing. Prentice Hall Professional Technical Reference, 2002.
[11] N. K. Sreelaja and G. A. V. Pai, "Swarm intelligence based key generation for text encryption in cellular networks," in Communication Systems Software and Middleware and Workshops, 2008. COMSWARE 2008. 3rd International Conference on, 2008, pp. 622-629.

[12] S. NK and G. A. V. Pai, "Design of Stream Cipher for Text Encryption using Particle Swarm Optimization based Key Generation., Journal of Information Assurance and Security ,30-41 2009

[13] R. A. Ali, "Random Number Generator based on Hybrid Algorithm between Particle Swarm Optimization (PSO) Algorithm and 3D-Chaotic System and its Application," Iraqi J. Inf. Technol., vol. 8, no. 3 pp. 1-20, 2018.

[14] A. A. Ghazi and F. H. Ali, "Robust and Efficient Dynamic Stream Cipher Cryptosystem," Iraqi J. Sci., vol. 59, no. 2C, pp. 1105-1114, 2018.

[15] H. C. Ong, S. L. Tilahun, and S. S. Tang, "A Comparative Study on Standard, Modified and Chaotic Firefly Algorithms," Goal Pertanika, p. 251, 2015.

[16] C. Blum and X. Li, "Swarm intelligence in optimization," in Swarm Intelligence, Springer, 2008, pp. $43-85$.

[17] A. T. S. Al-Obaidi, H. S. Abdullah, and Z. O. Ahmed, "Meerkat Clan Algorithm: A New Swarm Intelligence Algorithm," Indones. J. Electr. Eng. Comput. Sci., vol. 10, no. 1, pp. 354-360, 2018.

[18] A. Slowik and H. Kwasnicka, "Nature Inspired Methods and Their Industry Applications-Swarm Intelligence Algorithms," IEEE Trans. Ind. Informatics, vol. 14, no. 3, pp. 1004-1015, 2018.

[19] I. Fister Jr, M. Perc, S. M. Kamal, and I. Fister, "A review of chaos-based firefly algorithms: perspectives and research challenges," Appl. Math. Comput., vol. 252, pp. 155-165, 2015.

[20] X.-S. Yang and X. He, "Firefly algorithm: recent advances and applications," Int. J. Swarm Intell., vol. 1, no. 1, pp. 36-50, 2013.

[21] H. Wang, Z. Cui, H. Sun, S. Rahnamayan, and X.-S. Yang, "Randomly attracted firefly algorithm with neighborhood search and dynamic parameter adjustment mechanism," Soft Comput., vol. 21, no. 18, pp. 5325-5339, 2017.

[22] X.-S. Yang, "Nature-Inspired Metaheuristic Algorithms," Luniver Press. UK, pp. 242-246, 2008.

[23] B. Schneier, Applied cryptography: protocols, algorithms, and source code in $C$. john wiley \& sons, 2007. 
Journal of AL-Qadisiyah for computer science and mathematics Vol.10 No.3 Year 2018 ISSN (Print): 2074 - $0204 \quad$ ISSN (Online): 2521 - 3504

Mohammed .S/Nidaa .F

Appendix A

S1 and S2 are generated randomly as follows:

S1=[0xB2C420F8,033218BD4E,0x84590D94,0xDS1D3ASC,0xA3AB3D24,0X2A339E3D,0xFEE67A

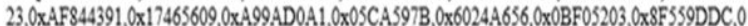

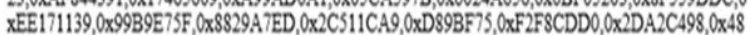

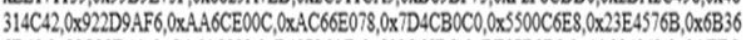

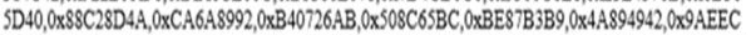

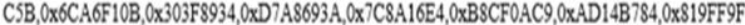

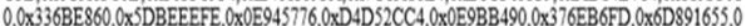
XD4078FEE, $0 x E 07401 E 7,0 x A 1 E 4350 C, 0 x A B C 78246,0 x 73409 C 02,0 \times 224704 A 1 F, 0 x 478 A B B 2 C, 0 x A 0$

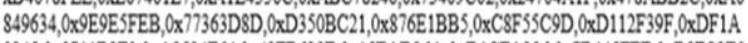
0245, 0x9711B3F0,0xA3334F64, 0x42FB629E,0x15EAD26A, 0xD1CFA296,0x7B445FEE,0xBSFC2EO D, 0x80168E15,0x0D7DEC9D, 0xCS581F55,0xBE4A2783,0xD27012FE, 0x33EA81CA,0xEBAA07D2 ,OX $44 F 5 D 41 D, 0 \times A B B 26 F A 6,0 \times 41 B 9 E A D 9,0 x A 48174 C 7,0 \times 1 F 3026 F 0,0 \times E F B A D D B E, 0 \times 387 E 9014,0$

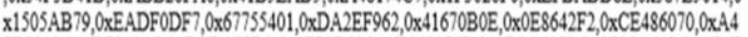
7D3312,0x4D7343A7,0xECDA58D0,0xIF79D536,0xD362376B,0x9D3A6023,0xC795A610,0xAE4D

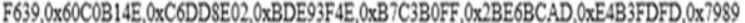

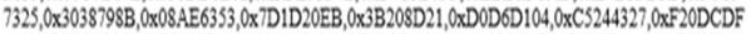

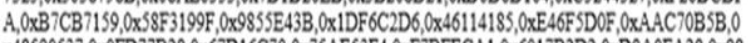

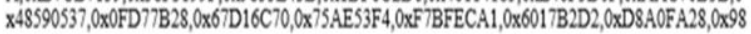

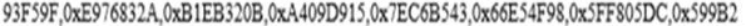

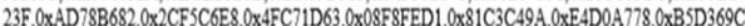

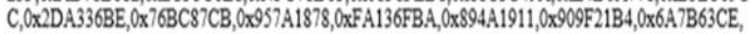
OXE28DD E4F177A,0XD17959B2,0x12AA6FFD, OxID39A8BE,077667FSAC,0xEDOCE165,0xF1658FDS,0x28

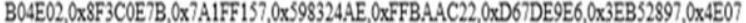
E855,0X87CE73F5, 0x8D046706, 0xD42D18F2, 0XE71B1727,0X33473B38,0xB37B24D5,0X381C6AE1 ,OXEาTD $6589,0 \times 6018 C B F, 0 \times 93$ CF $3752,0 \times 9 B 6 E A 235,0 \times 504 A 50 E 8,0 \times 464 E A 180,0 \times 86$ AFBESE, OXC C2D6AB0, $0 \times A B 91707 B, 0 \times 1 D B 4 D 579,0 \times F 9 F A F D 24,0 \times 2 B 28 C C 54,0 \times C D C F D 6 B 3,0 \times 68 A 30978,0 \times 4$ 3A6DFD7,0XC81DD98E, OXA6C2FD31,0x0FD07443,0XAFB400CC,0xSAF11A03,0X2647A909,0x24

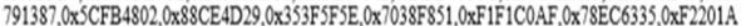
D1,0xDF 403361,0X4462DFC7,0xE22C5044,0x9C829EA3,0x43FD6EAE, OX7A42B3A7,0XSBFAAAE C, $0 \times 3$ EE $046833,0 \times 5789 D 266,0 \times 3069 C E 1 A, 0 \times F 115 D 008,0 x 4553$ AA9F, $033194 B E 09,0 \times B 4 A 9367 D, 0 x$ OA9DFEEC, Ox 7CA002D6,0X8E33A877,0X965E8183, 0XE1219370,0XIFA480CF, OXD3FB6FEF,0xED

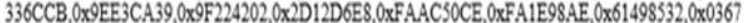

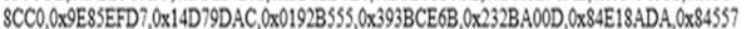
BA7,0x $68289948,0 \times 16690853,0 \times 414 A 3437,0 \times 7 B B 44897,0 \times 2315 B E 89,0 \times 7 A 01 F 224,0 \times 7056 A A S D, 0$ X121A3917,E3F47FA2, OXIF99DOAD, OX9BADS18B 1;

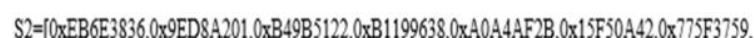

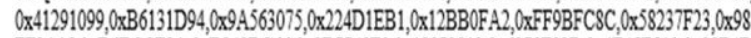
EF2A15,0xDGBCCF8A, $0 \times B 340 D C 66,0 \times 0 D 7743 F 0,0 \times 13372812,0 \times 6279 F 82 B, 0 \times 4 E 45 E 519,0 \times 98 B 4 B$

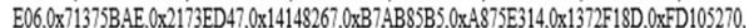

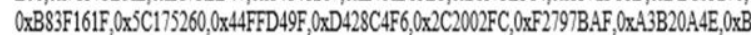

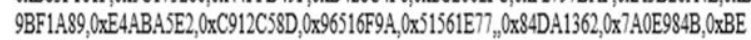

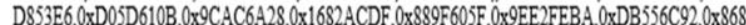

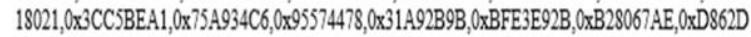
848,0x0732A22D, 0x840EF879,0x79FFA920,0x0124C8BB,0x26C75B69,0xC3DAAAC5,0xF1C871A D, 0x6C678B4D, ,x 46617752, OxAAE49354, 0xCABE8156,0x6D0AC54C,0x680CA74C, OxSCD82B3F,

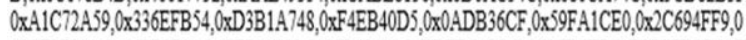
x 5 CE2F81A,0x469B9E34,0xCE74A493,0x OBB55111,0xEDED517C,0x1695D6FE, 0xE37C7EC7,0x57

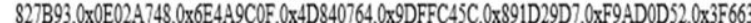

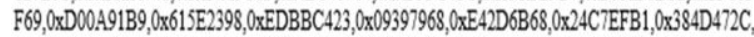

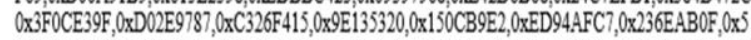

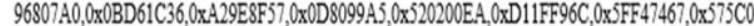
B39,0xOFC89690,0xB1FBACE8,0x7A957D16,0xB54D9F76,0x21DC77FB, 0x6DE85CF5,0xBFE7AE

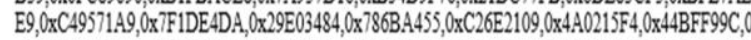
x 711A2414, 0xFDE9CDDD,0xDCE15B77,0x66D37887,0xF006CB92,0x27429119,0xF3789784,0x9B

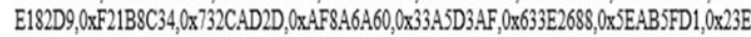
$60174,0 \times A C 27 A 7 C F, 0 x F=F C 5 A 0 E, 0 x C C 857 A 5 D, 0 \times 20 F B 7 B 56,0 \times 3241 F 4 C D, 0 x E 132 B 8 F 7,0 x 4 B B 3$

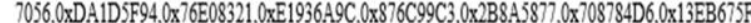

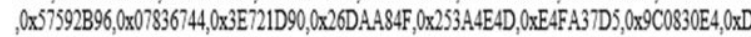

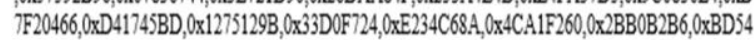

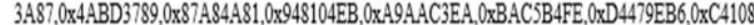

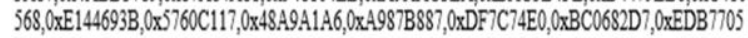

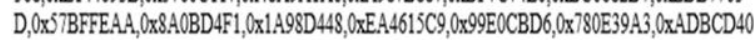
6.0xA94BC384,0xF7A81CAE,0xABS4ECD4,0x00DEF340,0x8E2329B8,0x23AF3.422,0x23C241FA,

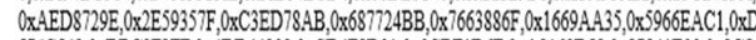
$574 C 543,0 \times D B C 3 F 2 F F, 0 \times 4 D D 44303,0 x C D 4 F 8 D 01,0 \times 0 C B F 1 D 6 F, 0 x A 8169059,0 \times 87841 E 00,0 \times 3$ C C5 15AD4,0x6E71F2E9,0X9FD4AFA6,0x474D0702,0x8B6AD73E,0xF5714E20,0xE608A352,0x2BF64

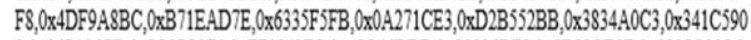

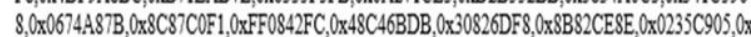
DE4844C3,0x296DF078,0xEFA46FEA,0x6CB98D67,0x6E959632,0xD5D3732F,0x68D95F19,0x43

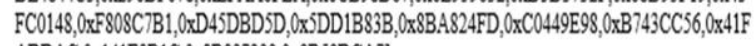
ADDAC, $, 0 x 141 E 9 B 1 C, 0 \times 8 B 937233,0 \times 9 B 99 D C A 7] ;$

$$
\begin{aligned}
& \text { تصميم مولا مفاتيح انسيابي بواسطة خوارزمية اليراعة } \\
& \text { نداء فليح حسن } \\
& \text { جامعة التكنولوجية } \\
& \text { كلية علوم الحاسوب }
\end{aligned}
$$

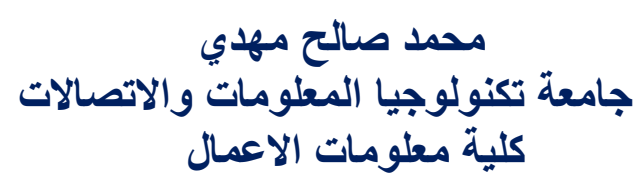

التشفير الانسيابي هو احدى طرق التشفير للبيانات المرسلة عبر الانترنت. التشفير الانسيابي ملائم في الاتصالات

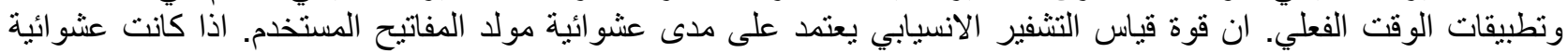

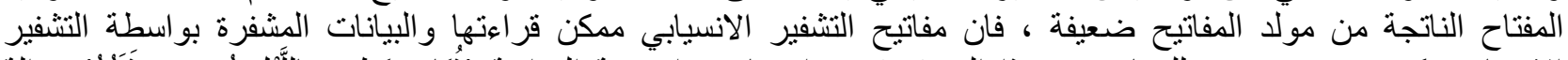

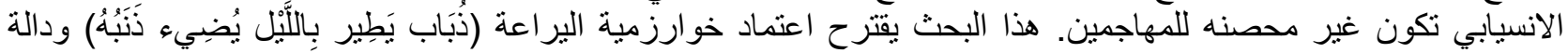

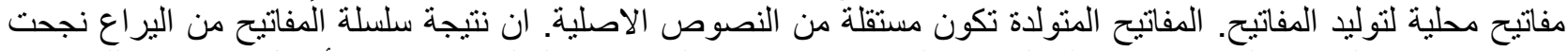

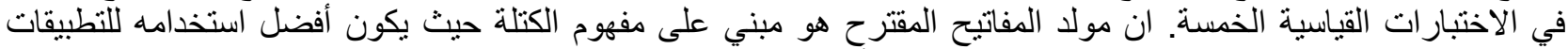

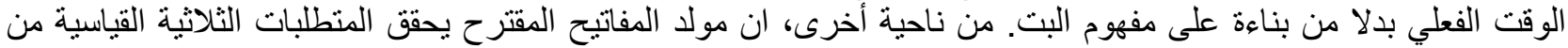

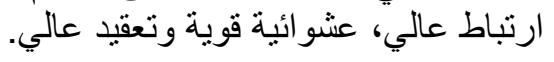

الكلمات المفتاحية: التشفير الانسيابي، مولد مفاتيح، خوارزمية الير اعة، دالة مفاتيح محلي . 